\title{
Alkyl polyglucoside vs. ethoxylated surfactant-based microemulsions as vehicles for two poorly water-soluble drugs: physicochemical characterization and in vivo skin performance
}

\author{
NATAŠA Z. BUBIĆ PAJIĆ ${ }^{1}$ \\ MARIJA N. TODOSIJEVIĆ 2 \\ GORDANA M. VULETA ${ }^{2}$ \\ NEBOJŠA D. CEKIĆ 3,4 \\ VLADIMIR D. DOBRIČIĆ ${ }^{5}$ \\ SONJA R. VUČEN ${ }^{6}$ \\ BOJAN R. ČALIJA ${ }^{2}$ \\ MILICA Ž. LUKIĆ ${ }^{2}$ \\ TANJA M. ILIĆ ${ }^{2}$ \\ SNEŽANA D. SAVIĆ ${ }^{*}$
}

${ }^{1}$ Department of Pharmaceutical Technology and Cosmetology Faculty of Medicine, University of Banja Luka, 78000 Banja Luka Bosnia and Herzegovina

${ }^{2}$ Department of Pharmaceutical Technology and Cosmetology Faculty of Pharmacy, University of Belgrade, 11221 Belgrade, Serbia

${ }^{3}$ Faculty of Technology, University of Niš, 16000 Leskovac, Serbia

${ }^{4}$ DCP Hemigal, 16000 Leskovac, Serbia

${ }^{5}$ Department of Pharmaceutical Chemistry, Faculty of Pharmacy University of Belgrade, Belgrade, Serbia

${ }^{6}$ School of Pharmacy, University

College Cork, Cork, Ireland
Two types of biocompatible surfactants were evaluated for their capability to formulate skin-friendly/non-irritant microemulsions as vehicles for two poorly water-soluble model drugs differing in properties and concentrations: alkyl polyglucosides (decyl glucoside and caprylyl/capryl glucoside) and ethoxylated surfactants (glycereth-7-caprylate/ caprate and polysorbate 80). Phase behavior, structural inversion and microemulsion solubilization potential for sertaconazole nitrate and adapalene were found to be highly dependent on the surfactants structure and HLB value. Performed characterization (polarized light microscopy, $\mathrm{pH}$, electrical conductivity, rheological, FTIR and DSC measurements) indicated a formulation containing glycereth-7-caprylate/caprate as suitable for incorporation of both drugs, whereas alkyl polyglucoside-based systems did not exhibit satisfying solubilization capacity for sertaconazole nitrate. Further, monitored parameters were strongly affected by sertaconazole nitrate incorporation, while they remained almost unchanged in adapalene-loaded vehicles. In addition, results of the in vivo skin performance study supported acceptable tolerability for all investigated formulations, suggesting selected microemulsions as promising carriers worth exploring further for effective skin delivery of model drugs.

Keywords: alkyl polyglucoside, ethoxylated surfactant, adapalene, sertaconazole nitrate, biocompatible microemulsion, in vivo skin irritation

Accepted September 18, 2017

Published online October 9, 2017

It is well known that microemulsions, as thermodynamically stable, optically isotropic colloidal systems, have several advantages, such as ease of manufacture, long-term stability, high solubilization capacity for lipophilic drugs and improved penetration of

\footnotetext{
* Correspondence; e-mail: snexs@pharmacy.bg.ac.rs
} 
both lipophilic and hydrophilic drugs through biological membranes, attributable to a continuously and spontaneously fluctuating interface of microemulsions, a high concentration gradient of solubilized drug, prospective surfactant interaction with lipid lamellae within the stratum corneum (SC), to mention just a few $(1,2)$. However, one of the main challenges in the development of microemulsions for topical application is avoidance of skin irritation, which usually arises from the high amount of surfactants in their formulation. To overcome this disadvantage, selection of surfactants with low skin irritation potential, such as sugar-based tensides, can be highly beneficial (1-3). For example, alkyl polyglucosides (APGs) are a relatively new class of mild, nonionic and non-ethoxylated biodegradable tensides, made from renewable raw materials, glucose and fatty alcohols (4) (structure is given in Fig. 1). Incorporation of such naturally derived surfactants, which are able to generate mild and nonirritant microemulsion systems, may be an advantage over conventionally used synthetic ethylene oxide-based surfactants $(2,3,5)$. However, most APGs are commercially available as complex mixtures, making their phase behavior studies challenging, since the commercial blends may differ in the carbon chain length, glycosylation number and stereochemistry $(4,5)$. In spite of a substantial amount of studies exploring APGs as surfactants for several types of applications, the literature describes few successful attempts dealing with the APG-based microemulsion systems as carriers for delivery of drugs or cosmeceuticals (2, 7-10). Thus, in the present work, we employed the so-called biocompatible surfactants for microemulsion formation, two of them belonging to the group of rarely used APGs, decyl glucoside (Planatacare 2000 UP, P2000) and caprylyl/ capryl glucoside (Plantacare 810 UP, P810), intending to compare them with their ethoxylated counterparts. For this purpose, ethoxylated surfactants (polysorbate 80 (P80) and glycereth-7-caprylate/caprate (Emanon EV-E, EV)) (Fig. 1) were also used for microemulsion fabrication and compared with APG-based microemulsion systems. While P80 represents a commonly used surfactant in microemulsion fabrication, the potential of EV in

a)

$$
\begin{aligned}
& \mathrm{CH}_{2} \mathrm{O}\left(\mathrm{CH}_{2} \mathrm{CH}_{2} \mathrm{O}\right)_{x} \mathrm{R} \quad \text { Sum of } \mathrm{x}, \mathrm{y} \text { and } \mathrm{z} \text { is } 7 \\
& \mathrm{CHO}\left(\mathrm{CH}_{2} \mathrm{CH}_{2} \mathrm{O}\right)_{y} \mathrm{R} \quad \mathrm{R}=\mathrm{H} \text { or R'- } \mathrm{CO}- \\
& \mathrm{CH}_{2} \mathrm{O}\left(\mathrm{CH}_{2} \mathrm{CH}_{2} \mathrm{O}\right)_{2} \mathrm{R} \quad \mathrm{R}^{\prime}=\text { Caprylic/Capric } \\
& \text { chain }
\end{aligned}
$$

b)

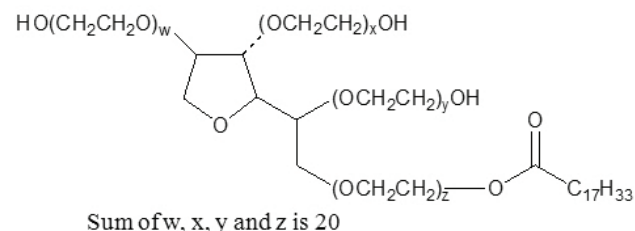

Fig. 1. Chemical structure of: a) Emanon EV-E, b) polysorbate 80, and c) APGs. c)

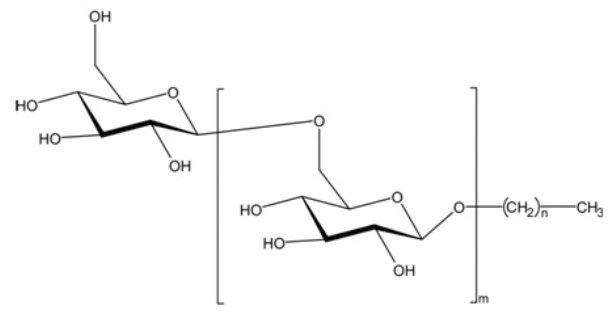

$\mathrm{m}$ - number of glucose units $\mathrm{n}$ - number of carbon atoms in the alkyl chain 
N. Z. Bubić Pajić et al.: Alkyl polyglucoside vs. ethoxylated surfactant-based microemulsions as vehicles for two poorly water-soluble drugs: physicochemical characterization and in vivo skin performance, Acta Pharm. 67 (2017) 415-439.

their formulation has been poorly explored. EV is a vegetable-based surfactant with low toxicity. It contains a high amount of monoester and shows a very high hydrophilic-lipophilic balance (HLB) value ( 17).

Besides, to extensively explore the possibility of employing each of the abovementioned surfactants in formulation of topical microemulsions, we selected two different model drugs with quite challenging properties: adapalene (ADA) and sertaconazole, in the form of nitrate salt (sertaconazole-nitrate, SN). ADA (Table I), a highly lipophilic third generation retinoid (synthetic analog of retinol), is commonly used as the first choice drug in acne therapy and in the treatment of photoaged skin (11). On the other hand, SN (Table I) is

Table I. Chemical structure and solubility of ADA and SN in various oils, surfactants and cosurfactants ${ }^{a}$

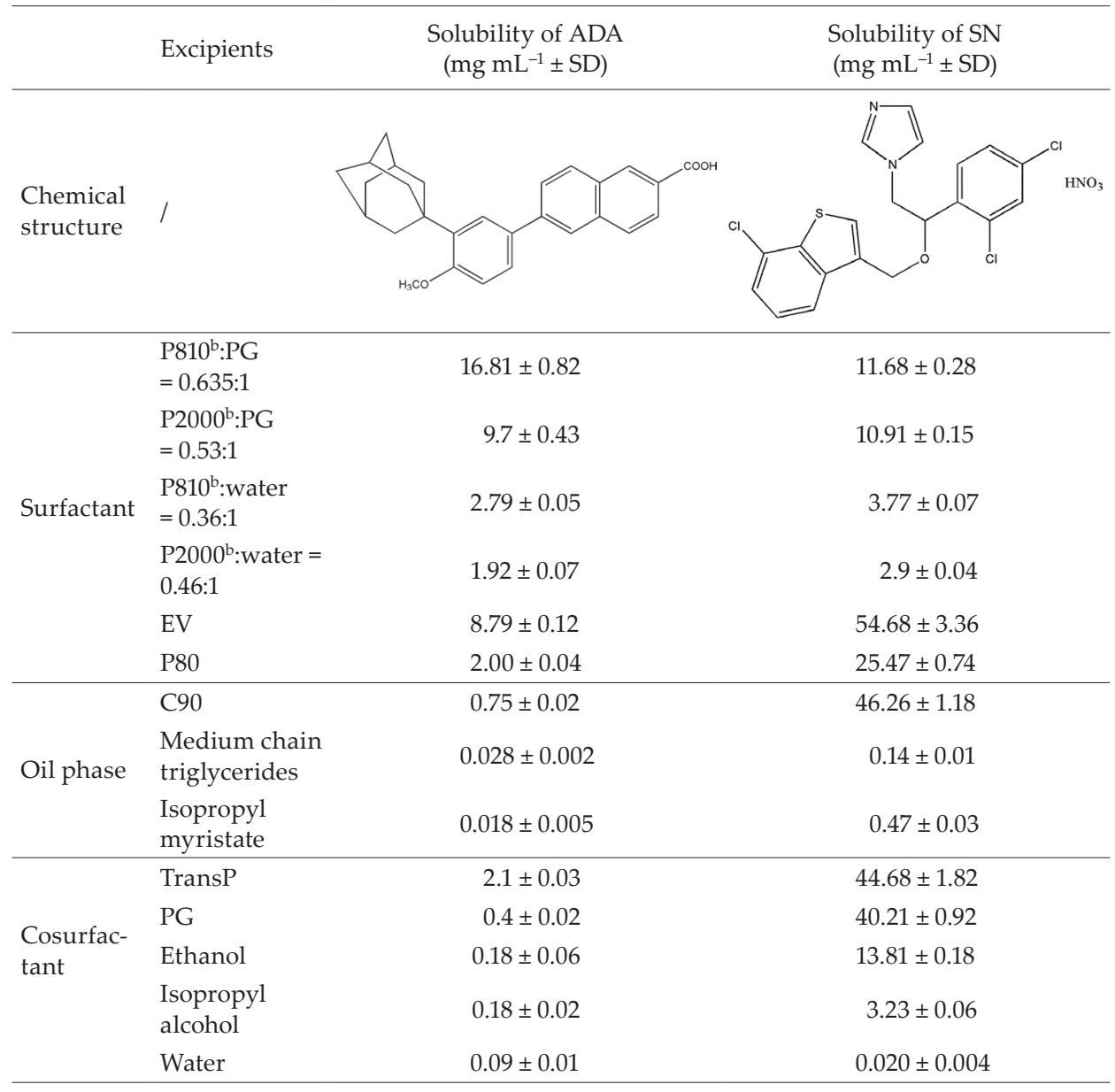

a Results are expressed as mean \pm SD of triplicate experiments

${ }^{\mathrm{b}}$ Only active substance regarded 
N. Z. Bubić Pajić et al.: Alkyl polyglucoside vs. ethoxylated surfactant-based microemulsions as vehicles for two poorly water-soluble drugs: physicochemical characterization and in vivo skin performance, Acta Pharm. 67 (2017) 415-439.

a broad spectrum imidazole antifungal agent, indicated for the treatment of superficial skin mycoses such as dermatophytosis, cutaneous candidiasis and seborrheic dermatitis of the scalp (12). Though both of them are poorly water-soluble drugs, they have diverse chemical structures (Table I) and, consequently, different physicochemical properties, which should be considered during the microemulsion formulation, such as dissociation characteristics and lipophilicity $\left(\mathrm{p} K_{\mathrm{a}} 4.23\right.$ and 7.26 and calculated $\log P 8.04$ and 6.20 for ADA and SN, respectively) $(13,14)$. In addition, ADA and SN strongly vary in their common therapeutic concentrations; while ADA is commercially available as a hydrogel, cream and lotion in a concentration of $0.1 \%(\mathrm{~m} / \mathrm{m})$, the therapeutic concentration of SN in various commercial topical dosage forms is much higher $(2 \%, \mathrm{~m} / \mathrm{m})$. However, application of such conventional vehicles is followed by limited topical bioavailability in the case of both drugs. Actually, the greatest challenge to their topical administration is how to reach viable epidermis and/or hair follicles in therapeutically valuable concentrations, at the same time avoiding the appearance of severe side effects, which may result from an inadequate vehicle formulation choice $(11,13,14)$. In this regard, microemulsion formulations based on non-irritating surfactants with adjusted inner structure appeared to be a valuable approach for topical delivery of ADA and SN as poorly soluble lipophilic entities, which were, to the best of our knowledge, scarcely evaluated in microemulsion systems up to now.

To be more precise, the main purpose of this work was to develop different well-tolerated, biocompatible colloidal vehicles for two model drugs of distinct physicochemical characteristics, ADA and SN, intended for topical administration. In this context, we were firstly interested in determining and comparing the pseudo-ternary phase behavior of different nonionic ethoxylated and newer non-ethoxylated surfactants with potentially improved skin tolerability and investigating how the structural differences and the HLB value of investigated surfactants affect their phase behavior. Since drug solubilization capacity and drug delivery potential of microemulsions depend on various factors, such as type of constituents, nanostructure of the system, physicochemical characteristics of the drug and consequently its localization (15), the structure and type of microemulsions was further investigated, using electrical conductivity, viscosity measurements and differential scanning calorimetry (DSC). In addition, solubilization studies were conducted in order to choose optimal vehicles with the lowest surfactant concentration for each investigated drug, followed by a comprehensive physicochemical characterization of the selected unloaded and drug-loaded microemulsions. Finally, in order to evaluate the skin irritation potential/skin tolerability of formulated microemulsions, an in vivo skin performance study, utilizing the biophysical methods coupled with an adequate study design, was performed.

\section{EXPERIMENTAL}

\section{Materials}

ADA was purchased from Beijing Mesochem Technology (Beijing, China) and SN from Shanghai Pengteng Fine Chemical (Shanghai, China). ADA reference standard (min. $99.4 \%$ ) was kindly donated by the Agency for Medicinal Products and Medical Devices, Bosnia and Herzegovina. SN reference standard was purchased from Sigma Aldrich (Steinheim, Germany). APGs (P2000 and P810) were a generous gift from BASF (Ludwig- 
N. Z. Bubić Pajić et al.: Alkyl polyglucoside vs. ethoxylated surfactant-based microemulsions as vehicles for two poorly water-soluble drugs: physicochemical characterization and in vivo skin performance, Acta Pharm. 67 (2017) 415-439.

shafen, Germany). In addition, EV was donated from Kao Chemicals (Barbera del Valles, Spain), while propylene glycol monocaprylate (Capryol 90, C90) and highly purified diethylene glycol monoethyl ether (Transcutol P, TransP) were gifts from Gattefosse (Lyon, France). Propylene glycol (PG) was purchased from Kemig (Zagreb, Croatia), P80, medium chain triglycerides and isopropyl myristate from Comcen (Belgrade, Serbia). HPLC grade water was obtained using a GenPure apparatus (TKA water purification system, Niederelbert, Germany). All other chemicals were of pharmaceutical grade and were used as received without purification.

\section{Solubility study and screening of excipients}

In order to find optimal microemulsion components, solubility studies of ADA and $\mathrm{SN}$ in water, various oils (C90, isopropyl myristate, medium chain triglycerides) and cosurfactants (ethanol, isopropyl alcohol, PG, TransP) were performed using the shake-flask method. In addition, to elucidate the possible distribution of ADA and SN in formulated microemulsions, solubility in surfactants and surfactant and cosurfactant mixtures $\left(S_{\text {mix }}\right)$ was determined. An excess amount of each drug was added to $5 \mathrm{~mL}$ of the sample in Erlenmeyer flasks and the resulting mixtures were continuously shaken for $48 \mathrm{~h}$, at a mixing rate of $300 \mathrm{rpm}$ using an IKA ${ }^{\circledR}$ KS 260 basic shaker (IKA ${ }^{\circledR}$ Werke GmbH \& Company KG Staufen, Germany). Afterwards, the samples were centrifuged at $3000 \mathrm{rpm}$ for 30 minutes to remove undissolved drug (Centrifuge Rotofix 32 A, Hettich, Tuttlingen, Germany). Aliquots of supernatant were filtered through a $0.22 \mu \mathrm{m}$ membrane filter $\left(\right.$ Chromafil $^{\circledR}$ Xtra PTFE-20/25, Macherey-Nagel, Düren, Germany). In the case of ADA, its concentration in the supernatant was determined using liquid chromatography-tandem mass spectrometry (LC-MS/MS) method. The analysis was performed on a UHPLC chromatograph ACELLA (Thermo Fisher Scientific Inc., USA), coupled to a triple quadrupole mass spectrometer TSQ Quantum Access MAX (Thermo Fisher Scientific Inc.) with heated electrospray ionization (HESI) interface. The column was Zorbax Eclipse XDB-C18 $(150 \mathrm{~mm} \times 4.6 \mathrm{~mm}, 5 \mu \mathrm{m}$ particle size) from Agilent Technologies (USA). Mobile phase was acetonitrile $/ 20 \mathrm{mmol} \mathrm{L}^{-1}$ ammonium acetate $=70: 30(\mathrm{~V} / \mathrm{V})$, flow rate was $0.8 \mathrm{~mL} / \mathrm{min}$ and column temperature was set to $25{ }^{\circ} \mathrm{C}$. Injection volume was $10 \mu \mathrm{L}$. The monitored SRM transition was $411 \rightarrow 367$. On the other hand, SN solubility in the supernatant was determined by analyzing the filtrate spectroscopically, after proper dilution with methanol, at $260 \mathrm{~nm}$ (UV-1800 spectrophotometer, Shimadzu, Japan). Experiments were performed in triplicate, at room temperature.

\section{Phase behavior and construction of pseudo-ternary phase diagrams}

In order to formulate biocompatible vehicles with optimal solubilization capacity for each drug, emulsification capability of APGs was further investigated and compared with the phase behavior of synthetic ethoxylated surfactants EV and P80. Among the surfactants chosen, two have similar HLB values of around 15 (P810 and P80), whereas HLB value of P2000 is slightly lower ( 13) and that of EV slightly higher $(\sim 17)(16,17)$. For the purpose of phase behavior investigations, pseudo-ternary phase diagrams were constructed using the water titration method at room temperature. First, a precise amount of each surfactant was mixed with the required amount of cosurfactant, in a mass ratio $1: 1\left(K_{\mathrm{m}}\right.$ ratio 1:1), using a magnetic stirrer, and this ratio was kept constant throughout the phase 
N. Z. Bubić Pajić et al.: Alkyl polyglucoside vs. ethoxylated surfactant-based microemulsions as vehicles for two poorly water-soluble drugs: physicochemical characterization and in vivo skin performance, Acta Pharm. 67 (2017) 415-439.

behavior investigation. It should be noted that both commercial samples of APGs used in this study contained a certain amount of water. According to the manufacturer's specification, P2000 contains $53 \%$ of pure surfactant and $47 \%$ water, while P810 is a mixture consisting of $63.5 \%$ surfactant and $36.5 \%$ water. Hence, the real $K_{\mathrm{m}}$ ratios for these systems were 0.530:1 and 0.635:1 for P2000 and P810, respectively. The ratio of oil phase to $S_{\text {mix }}$ was varied as 1:9, 2:8, 3:7, 4:6, 5:5, 6:4, 7:3, 8:2, and 9:1. The mixture of oil and $S_{\text {mix }}$ was titrated with small aliquots of water under vigorous agitation (Vortex Mixer, Velp Scientifica, Italy). After equilibration for 15 minutes, the mixture was assessed by visual characterization. Samples that remained homogenous, single-phase, low viscous and transparent after vigorous vortexing were assigned to a monophasic area in the phase diagram. Turbidity was considered as an indicator of phase separation in the system. Boundaries of the microemulsion region were determined by observing the changes in sample appearance from transparent to turbid and then calculating the percent of incorporated water phase.

\section{Selection of formulation and preparation of samples}

From the constructed phase diagrams, selection of microemulsions was done based on the determination of solubilization capacity of numerous formulations along the selected dilution line, which was chosen based on the capacity for water solubilization, while keeping the surfactant concentration at minimum level. Microemulsion systems were prepared by mixing the oil phase and $S_{\text {mix }}$, and thereafter water phase was added precisely under magnetic stirring at room temperature. The systems were equilibrated by gentle magnetic stirring for $30 \mathrm{~min}$. Drug-loaded formulations were prepared by adding the drug into pre-weighted microemulsion vehicles under continuous mixing with a magnetic stirrer (Falc, Italy) to give the final concentration of $0.1 \%(\mathrm{~m} / \mathrm{m})$ and $2 \%(\mathrm{~m} / \mathrm{m})$ of ADA and SN, respectively. The samples were kept in well-closed glass containers and stored at room temperature for $48 \mathrm{~h}$ to equilibrate before further characterization.

Microemulsions with sufficient solubilization capacity for ADA or SN were identified based on their visual appearance and optical behavior using polarization microscopy, as transparent and isotropic systems, respectively, without any physical change (e.g., undissolved drug particles, blurriness). In addition, saturated solubility of each drug was determined in selected formulations in order to test the correlation between microemulsion nanostructure upon dilution and maximum solubilization capacity. An excess drug amount was added into the selected formulation and stirred continuously for $48 \mathrm{~h}$ at a mixing rate of $300 \mathrm{rpm}$, at room temperature, to reach equilibrium. After centrifugation (3000 rpm for 30 minutes), the supernatant was filtered through a $0.22 \mu \mathrm{m}$ membrane filter and the concentration of the drug was determined as previously described.

\section{Interfacial tension measurement}

To investigate the potential amphiphilic nature of ADA and to deeper explore the drug interaction with microemulsion constituents, the dynamic interfacial tension between water and oil as well as water and oil containing ADA $\left(0.5 \mathrm{mg} \mathrm{mL}^{-1}\right)$ were measured in the time range from 1 to $900 \mathrm{~s}$ using a drop profile analysis tensiometer PAT1 (SINTERFACE Technologies, Germany) at room temperature. Oil drops were formed the tip of the capillary immersed into a cuvette filled with ultra-pure water. Each measurement was repeated five times. 
N. Z. Bubić Pajić et al.: Alkyl polyglucoside vs. ethoxylated surfactant-based microemulsions as vehicles for two poorly water-soluble drugs: physicochemical characterization and in vivo skin performance, Acta Pharm. 67 (2017) 415-439.

\section{Microemulsion characterization}

Polarization microscopy. - Optical isotropy of both unloaded and drug-loaded transparent vehicles was evaluated using cross-polarized light microscopy (Olympus BX51-P, Olympus Corporation, Tokyo, Japan). Polarization microscopy is a useful technique for distinguishing isotropic microemulsions from liquid-crystalline phases (8), by observing whether the sample rotates the plane of polarized light. Microemulsions, as transparent, monophasic systems, do not interfere with polarized light, so the field of the view remains dark (18).

pH analysis. - The $\mathrm{pH}$ values of microemulsions were evaluated using a $\mathrm{pH}$ meter (HI9321, Hanna Instruments, Portugal). Experiments were performed in triplicate for each sample.

Electrical conductivity measurements. - Electrical conductivity measurements were done at room temperature using a SensION EC71 instrument (Hach Company, Loveland, Colorado) with a cell constant of $1.0 \mathrm{~cm}^{-1}$. To evaluate the internal structure of the systems, the water phase was added dropwise into the initial $S_{\text {mix }}$ :oil $=8: 2$ mixture and conductivity of the obtained samples was checked after equilibrium was attained. A conventional way to check conductivity in nonionic microemulsions is to add a small concentration of salt into the water phase (19). Hence, the aqueous phase used was a $0.9 \%(\mathrm{~m} / \mathrm{V})$ sodium chloride solution. However, addition of electrolytes was not necessary in the case of APGs because they contain electrolytes derived from the production process (5).

Rheological measurements. - Rheological properties of the prepared samples were evaluated using a DV-III ULTRA Programmable Rheometer \& Rheocalc software v.4.3 (Brookfield Engineering Laboratories, Middlesboro, USA), coupled with a cone and plate measuring device at $20 \pm 1{ }^{\circ} \mathrm{C}$. Measurements were performed at shear rates ranging from 75 to $750 \mathrm{~s}^{-1}$, for both up and down curves. The obtained flow curves were evaluated by fitting the experimental data to Newtonian, Bingham and Ostwald models. The apparent viscosity $(\eta)$ of selected formulations was measured in triplicate at a shear rate of $375 \mathrm{~s}^{-1}$.

DSC measurements. - To investigate the physical state of water in non-ionic microemulsions, and thus obtain a further insight into the internal structure and type of prepared microemulsions, low-temperature DSC was employed. DSC cooling curves were obtained using a Mettler Toledo DSC 1, STAR ${ }^{\mathrm{e}}$ System (Mettler Toledo AG, Analytical, Switzerland). Approximately $10 \mathrm{mg}$ of microemulsion samples were weighed into aluminum pans and quickly sealed to prevent water evaporation from the samples. Simultaneously, an empty hermetically sealed pan was used as reference. Blank microemulsion samples were exposed to a temperature range from 25 to $-60{ }^{\circ} \mathrm{C}$ (scan rate: $5^{\circ} \mathrm{C} \mathrm{min}{ }^{-1}$ ) under a constant nitrogen flow $\left(50 \mathrm{~mL} \mathrm{~min}^{-1}\right)$.

FTIR spectroscopy. - To detect any possible interaction between the drug and the different components used, the FTIR spectra of the selected unloaded and drug-loaded microemulsions were obtained using a BOMEM Hartmann \& Braun MB-Series FTIR spectrophotometer (ABB Bomem Inc., Canada). Samples of pure drugs were prepared prior to FTIR studies using the potassium bromide (KBr) pastille method. For the microemulsion sample preparation, $10 \mu \mathrm{L}$ of microemulsion was placed on a KRS-5 plate and dried in vacuum. The FTIR spectra of all samples were recorded between 4000 and $400 \mathrm{~cm}^{-1}$, using 10 scans for each spectrum, at a resolution of $4 \mathrm{~cm}^{-1}$. 
N. Z. Bubić Pajić et al.: Alkyl polyglucoside vs. ethoxylated surfactant-based microemulsions as vehicles for two poorly water-soluble drugs: physicochemical characterization and in vivo skin performance, Acta Pharm. 67 (2017) 415-439.

\section{In vivo skin performance of selected microemulsions}

Considering that one of major drawbacks of microemulsion formulations is the ability to induce skin irritation $(1,3)$, it is highly desirable to assess the skin irritation potential during the development of microemulsions. Therefore, biophysical techniques, coupled with an adequate study design, were used not only to gain information about skin performance of selected microemulsions but the skin irritation potential as well.

In our study, 10 healthy volunteers ( $29.1 \pm 5.7$ years old $)$ with no history of skin diseases or allergic skin reactions were recruited to participate in a short-term 24-hour in vivo skin occlusion study. Prior to the study, they were first comprehensively informed about the study protocol and then they signed written consents. They were not allowed to use any cosmetic or pharmaceutical product on investigated skin sites $24 \mathrm{~h}$ before the beginning and during the study. Further, they were not allowed to consume nicotine and caffeine beverages $3 \mathrm{~h}$ before each measurement. The study was conducted in accordance with the Declaration of Helsinki and was approved by the local Ethical Committee on Human Research (University of Belgrade, Faculty of Pharmacy, Serbia).

Three test sites $(2 \times 2 \mathrm{~cm})$, at the volar aspects of each forearm, were precisely delineated using a cardboard template. Two areas were treated with investigated microemulsions and the third site was used as untreated control, one with occlusion (NCO) and the other without occlusion (NC). Samples were applied in the amount of $60 \mu \mathrm{L}$ and spread vigorously with a rubber glove. Afterwards, treated sites and the NCO test site were covered with Parafilm ${ }^{\circledR}$ (Pechiney Plastic Packaging, Inc., Wisconsin, USA) and cotton adhesive tape Sensifix ${ }^{\circledR}$ (Serbia) to create occlusion. Volunteers were instructed to remove the occlusion 24 hours after it was applied.

The assessed biophysical skin parameters were: erythema index (EI), stratum corneum hydration level (SCH) and transepidermal water loss (TEWL). All measurements were conducted with Courage\&Khazaka Electronic $\mathrm{GmbH}$ (Cologne, Germany) instruments: Mexameter ${ }^{\circledast}$ MX 18, Cutometer ${ }^{\circledast}$ MPA 580 with an integrated Corneometer ${ }^{\circledR} \mathrm{CM} 825$ and Tewameter ${ }^{\circledR}$ TM 210, respectively. First, initial measurements (basal values) were conducted prior to sample application and subsequent measurements were performed 3 hours upon the removal of occlusions. Volunteers spent 30 minutes in the study room before each measurement in order to adapt to the temperature and humidity conditions. All measurements were conducted in accordance with the published guidelines and studies (20-22).

\section{Statistical analysis}

Whenever applicable, results were presented as mean \pm standard deviation (SD). Statistical analysis was performed using Student's $t$-test or one-way analysis of variance (ANOVA), followed by post hoc Tukey's test, depending on the nature of the data (PASW Statistics version 18.0, SPSS Inc., USA). Statistical significance was set at $p<0.05$.

\section{RESULTS AND DISCUSSION}

\section{Screening of excipients}

Among the investigated oils (Table I), the solubility of ADA as well as of SN was found to be the highest in $\mathrm{C} 90$ ( 0.75 and $46.26 \mathrm{mg} \mathrm{mL}^{-1}$, respectively). This result was somehow 
N. Z. Bubić Pajić et al.: Alkyl polyglucoside vs. ethoxylated surfactant-based microemulsions as vehicles for two poorly water-soluble drugs: physicochemical characterization and in vivo skin performance, Acta Pharm. 67 (2017) 415-439.

expected, taking into account that the used oil phase C90 had surface active properties. Thus, C90 was selected as the oil phase throughout the overall course of further experiments. As can be seen in Table I, in the case of cosurfactants, TransP showed the highest potential to dissolve both drugs, followed by PG. Therefore, TransP was selected as cosurfactant. However, using TransP in combination with both APGs as surfactants $\left(K_{\mathrm{m}}\right.$ ratio 1:1) did not result in microemulsion formation. Therefore, in both APG-based formulations, PG was used as a cosurfactant instead of TransP. Other advantages of using 1,2-alkanediols are their good co-solvent properties and lower toxicity compared to the corresponding alkanols. Incorporation of 1,2-alkanediols may also improve skin penetration of the drug, even from conventional vehicles $(2,5)$.

\section{Phase behavior and construction of pseudo-ternary phase diagrams}

Based on the results of solubility, in the current study, two APG-based pseudo-ternary phase diagrams were constructed for the system C90/APG-PG/water, used as APGs P2000 and P810 (Figs. 2a and b). As can be seen, we have proven that formulation of microemulsions with APGs is quite difficult, since the obtained isotropic area was narrower compared to ethoxylated surfactants (pseudo-ternary phase diagrams shown in Figs. 2c and d), which is in line with the literature data $(4,23)$, probably because of lower oil solubility of APGs. In addition, the use of certain cosurfactants is recognized as being helpful for further reducing the interfacial tension, whilst increasing the fluidity of the interface, and for adjusting the HLB and spontaneous curvature of the interface by changing surfactant partitioning characteristics. Also, a cosurfactant may influence the solubility properties of both aqueous and oil phases for drugs due to its distribution between them (3). Hence, many experimental studies have been focused on the increase of oil solubility of APGs by using cosurfactants (alcohol or other surfactants). Anyway, the phase behavior of P2000 and P810 systems was found to be different, which is in line with the results obtained for other APGs $(8,23)$. The monophasic area was wider when P810 was used as a surfactant in comparison with the microemulsion region obtained with P2000. Indeed, the isotropic area was wider when a surfactant with shorter chain length was used. This is in accord with the previously reported findings $(18,24)$ where emulsion capacity of the other sugarbased surfactants was decreased as the hydrocarbon chain length increased, i.e., the surfactant with a short chain length was able to bind more water compared to the surfactant with a longer tail. Indeed, with increasing the tail length, the flexibility of the interfacial film becomes limited, making it more rigid (18). Therefore, the chemical structure of the APGs and, consequently, chain stiffness had probably some influence on the packaging of surfactant molecules at the interface, causing the differences between the presented phase diagrams. Although the same phenomenon, i.e., restriction of a total monophasic area by increasing the chain length, was detected for other sugar based surfactants such as sucrose esters $(18,24)$, it should be noted that most of the aforementioned sucrose esters had the same HLB values. Hence, in our case, the observed difference in phase diagrams could not be related only to the surfactant chain length, but also to the HLB values of the selected APGs (P2000 has HLB around 13 and P810 around 15-16) $(16,17)$ due to the different degrees of polymerization and their anomeric purity $(7,8,23)$. To be more precise, the increasing number of sugar units in their molecules (i.e., degree of polymerization) makes APGs more hydrophilic and thus less soluble in the oil phase (21). It should be born in mind that the complexity of APGs, as naturally derived compounds, has already been reported (6), 
a)

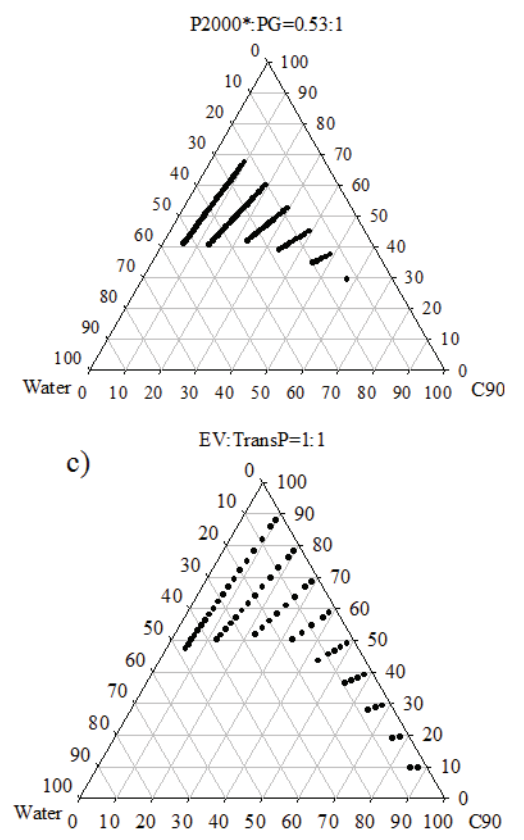

b)

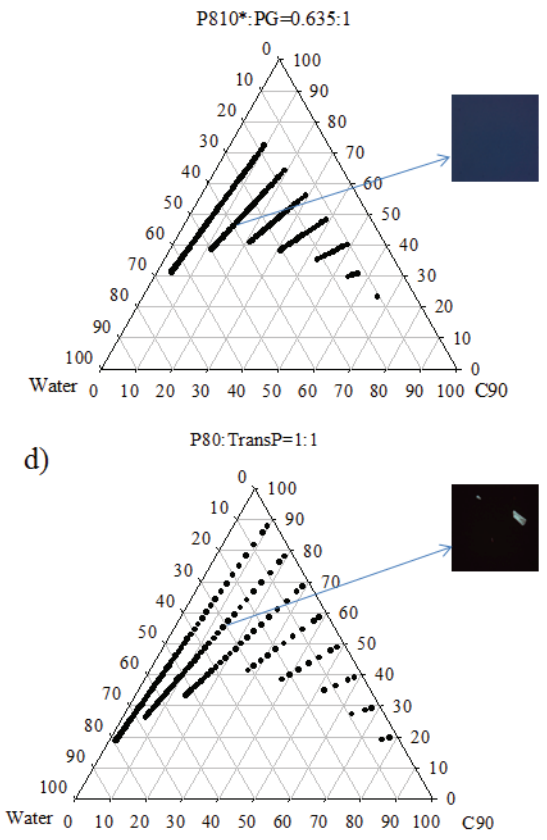

Fig. 2. Pseudo-ternary phase diagrams of the microemulsion composed of: a) C90/P2000*-PG/water, b) C90/P810*-PG/water, c) C90/EV-TransP/water, and d) C90/P80-TransP/water, 3b inset). Photomicrograph of APG-2 microemulsion, and 3d inset) photomicrograph of ME-P microemulsion with SN crystals, obtained using polarized light microscopy.

${ }^{*}$ Only active substance regarded

making microemulsion formulation even more difficult. Because both APGs used in this study have the average degree of polymerization of 1.5, their phase behavior mostly depends on the alkyl chain length and their anomeric configuration. Further, it should be also noted that P2000 and P810, as commercial mixtures, contain approximately $53 \%(\mathrm{~m} / \mathrm{m})$ of decyl glucoside and $63.5(\mathrm{~m} / \mathrm{m})$ of caprylyl/capryl glucoside, respectively. Hence, in the case of P810, the wider monophasic region may be ascribed not only to the shorter chain length of a part of the blend, but also to the higher content of surfactant molecules compared to the corresponding P2000-based system.

On the other hand, the phase behavior study of the ethoxylated surfactants revealed that P80 had higher water solubilization capacity compared to EV (Figs. 2c and d). Broader microemulsion area in the P80-based diagram (Fig. 2d) could be ascribed to the size of hydrophilic and lipophilic groups of the solubilizer as well as to its HLB value. Namely, it was previously demonstrated for alkyl polyoxyethylene that, when the hydrophilic and/or hydrocarbon chain length is larger (Figs. 1a and b), a larger single-phase area could be expected (25). However, these findings are different from those obtained for sugar surfactants $(7,18,24)$, making phase behavior investigation more complex. In addition, in most cases, the investigated sugar surfactants had the same head group as well as HLB values 
N. Z. Bubić Pajić et al.: Alkyl polyglucoside vs. ethoxylated surfactant-based microemulsions as vehicles for two poorly water-soluble drugs: physicochemical characterization and in vivo skin performance, Acta Pharm. 67 (2017) 415-439.

$(18,24)$ and, hence, the phase behavior was dependent on the hydrophobic chain length only. In contrast, alkyl polyoxyethylenes usually differ in sizes of both parts of the molecule, i.e., the hydrophilic and the lipophilic ones, resulting in different HLB values. It is well known that for optimal solubilization capability, a mixture of surfactant and cosurfactant should have a suitable HLB value, within the range required for the preparation of microemulsions $(3,26)$. In our case, both APG (P810) and ethylene oxide-based (P80) surfactants, which produced a wider monophasic area, had HLB values around 15 . However, in order to produce a larger microemulsion area, besides the optimal HLB of a surfactant, it is important to choose an adequate oil to achieve proper packaging of surfactant molecules at the oil-water interface (24). To summarize, while an increase in the hydrophobic part of sugar surfactant molecules leads to a decrease in total monophasic area, in the case of alkyl polyoxyethylenes the situation is quite opposite. In addition, we demonstrated that EV can be used as a surfactant for microemulsion fabrication, although its emulsification capability was slightly lower when compared to P80. However, higher solubility of the selected model drugs in EV may be considered advantageous (Table I). This may allow the concentration of the surfactant to be reduced in the final drug-loaded formulation, which in turn increases the biocompatibility of the microemulsion system $(15,25,26)$.

For further studies, we selected, irrespective of surfactant type, the water dilution line $S_{\text {mix }}: \mathrm{O}=8: 2$, because water solubilization was slightly lower than along the line $S_{\text {mix }}: \mathrm{O}=9: 1$, but good enough for investigation of solubilization capacity. Also, as previously reported $(1,26)$, the enhanced skin penetration and maximum flux in dermal drug delivery are not usually obtained with formulations that contain maximum surfactant concentration.

\section{Characterization of colloidal microstructure}

Electrical conductivity. - Electrical conductivity $(\kappa)$ was measured as a function of the mass ratio of aqueous phase $\left(\Phi_{\mathrm{w}}\right)$ along the dilution line $S_{\text {mix }}: \mathrm{O}=8: 2$ (Figs. 3a and b). As already mentioned, the commercially available investigated APGs represent surfactant molecules in a mixture with water. Therefore, variation of the system composition along the dilution line causes a change in the amount of APG molecules and thus the number of charge carriers, involving altered $\kappa$ values of microemulsions as well. Hence, the obtained values have to be corrected by dividing $\kappa$ by the surfactant fraction, resulting in corrected values $\left(\kappa_{\mathrm{a}}\right)(5)$. The $\kappa_{\mathrm{a}}$ values plotted versus $\Phi_{\mathrm{w}}$ (which is the sum of water already present in the surfactant and water added) in APG-based microemulsions are depicted in Fig. 3a. Obviously, $\kappa_{\mathrm{a}}$ continuously increased with $\Phi_{\mathrm{w}}$ increase, regardless of the surfactant used. In spite of similar trends, in the case of the system containing P810, the $\kappa_{\mathrm{a}} v \mathrm{~s} . \Phi_{\mathrm{w}}$ curve can be divided into three parts with different slopes, which may be related to the existence of three regions differing in their inner nanostructures (Fig. 3a). Low $\kappa_{\mathrm{a}}$ values were observed with less than $20 \%(\mathrm{~m} / \mathrm{m})$ of water (the first region), which may imply the existence of water in oil (w/o) microemulsions. The rise in conductivity with further addition of water (at $\sim 20-32 \%, \mathrm{~m} / \mathrm{m}$ ) suggested an increase in the interlinking process of aqueous droplets, resulting in the formation of clusters and therefore appearance of bicontinuous structures (the second region). When $\Phi_{\mathrm{w}}$ exceeded $32 \%(\mathrm{~m} / \mathrm{m}), \kappa_{\mathrm{a}}$ rose rapidly until the system became turbid, because the number of water channels and clusters increased and the surfactant head groups became saturated with water. Because the second change in the slope at $32 \%$ $(\mathrm{m} / \mathrm{m})$ of water is less visible, and due to high values of conductivity for the isotropic systems in the third region (with $\Phi_{\mathrm{w}}$ above $32 \%, \mathrm{~m} / \mathrm{m}$ ), only the beginning of transition from 
a)

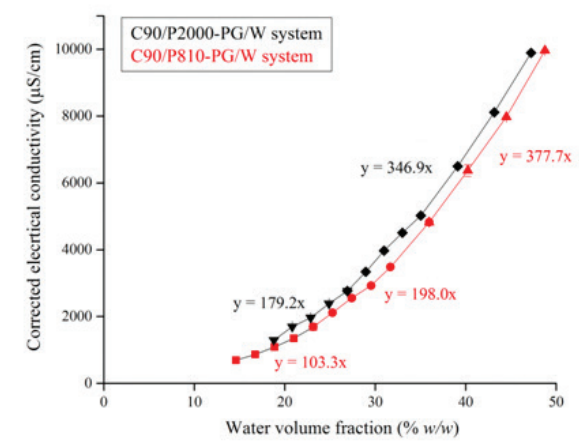

c)

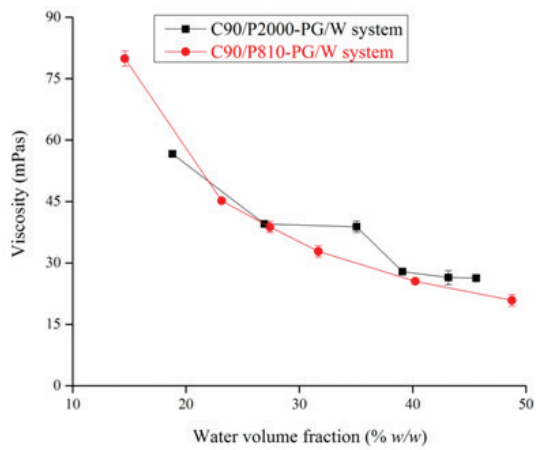

b)

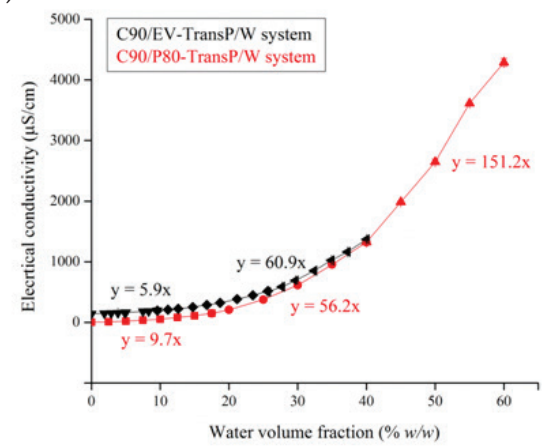

d)

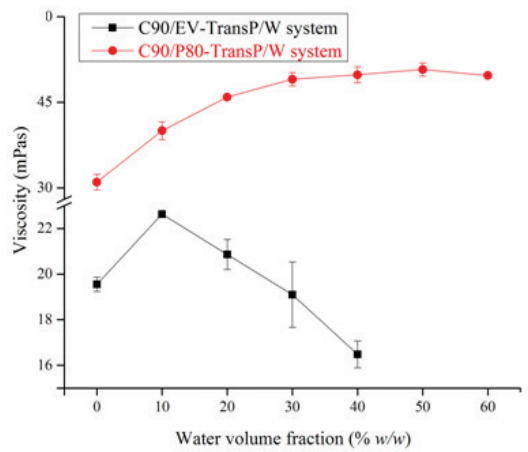

Fig. 3. Electrical conductivity $(\kappa)$ as a function of the water volume fraction $\left(\Phi_{\mathrm{w}}\right)$ for: a) C90/APG-PG/ water, and b) C90/ethoxylated surfactant-TransP/water microemulsions, and apparent viscosity as a function of the water volume fraction for: c) C90/APG-PG/water and d) C90/ethoxylated surfactantTransP/water microemulsions along the dilution line $S_{\text {mix }}: \mathrm{O}=8: 2$.

a bicontinuous to inverted bicontinuous structure may be presumed (10). Hence, in this third region, the o/w structure was not completely developed. For more detailed explanation of such phase transitions, we employed the percolation theory (27). In the percolation model, the $\kappa$ remains low up to a certain $\Phi_{w}$ due to the non-conducting nature of the continuous phase of the w/o microemulsion system (19). However, after reaching and exceeding the percolation threshold $\left(\Phi_{\mathrm{p}}\right)$, some of conductive aqueous droplets begin to contact each other and form water clusters, which generate conductive paths leading to a rapid increase in conductance from almost zero to much higher values (27). Therefore, the obtained $\Phi_{\mathrm{p}}$ value was around $20 \% \mathrm{~m} / \mathrm{m}$ for the system C90/P810-PG/water, as presented in Fig. 3a, suggesting that microemulsions with $\Phi_{\mathrm{w}}$ above $20 \%(\mathrm{~m} / \mathrm{m})$ had a bicontinuous structure, whereas w/o microemulsions were obtained with less than $20 \%(\mathrm{~m} / \mathrm{m})$ of water. On the other hand, no indication of the phase transition from w/o to bicontinuous microemulsions in the P2000-based system was noticed. The reason for such behavior may be the fact that P2000, as a commercial product, contains around $47 \%(\mathrm{~m} / \mathrm{m})$ of water and it might be possible that the bicontinuous structure was obtained directly by mixing $S_{\text {mix }}$ with the oil phase. These results indicate that APGs are not good surfactants for formula- 
N. Z. Bubić Pajić et al.: Alkyl polyglucoside vs. ethoxylated surfactant-based microemulsions as vehicles for two poorly water-soluble drugs: physicochemical characterization and in vivo skin performance, Acta Pharm. 67 (2017) 415-439.

tion of w/o microemulsion vehicles selected from the dilution line $S_{\text {mix }}: \mathrm{O}=8: 2$ due to the presence of water in commercially available APGs. Thus, the obtained bicontinuous systems can hardly be used as carriers for drugs with high oil solubility (such as sertaconazole), whereas the solubilization of mesogenic drugs at the interfacial film would be facilitated $(3,18,25)$. However, we do not exclude the possibility of realizing the oil continuous microstructure of samples selected from other dilution lines having the potential to solubilize drugs of high lipophilicity. Because skin penetration of lipophilic and hydrophilic drugs seems to be more pronounced with bicontinuous and o/w vehicles than oil continuous microemulsions $(1,14,19,32)$, no attempts to dissolve model drugs in formulations with a presumed w/o microstructure were made in this study.

In contrast, in both systems containing ethoxylated surfactants, typical percolative behavior could be clearly observed. As can be seen, in the investigated systems $\kappa$ increased continuously as $\Phi_{\mathrm{w}}$ increased (Fig. 3b). Respective conductivity profiles of C90/ethoxylated surfactant-TransP/water systems were different in comparison with APG-based formulations. The curve representing the dependence of $\kappa$ on $\Phi_{\mathrm{w}}$ in both ethoxylated surfactant containing systems showed that there was a change in system structure at $\Phi_{\mathrm{w}}$ around $10 \%$ $(\mathrm{m} / \mathrm{m})$, since the turning point on the curve from linear to non-linear represents an indicator of $\Phi_{\mathrm{p}}$ and corresponded to the structural inversion from w/o to bicontinuous microemulsions (19). However, in the case of the P80-containing system, conductivity measurements revealed regions with three different slopes, suggesting $\mathrm{o} / \mathrm{w}$ nanostructure above $55 \%(\mathrm{~m} / \mathrm{m})$ of water added (Fig. 3b). At that point, polar head groups of P80 were exposed to the continuous phase and became saturated with water, leading to maximum values of $\kappa$. Therefore, conductivity results of APGs and ethoxylated surfactants supported our assumptions regarding their different behavior and their potential to form microemulsions with different inner structures. To confirm electrical conductivity results, microemulsions were further characterized for their rheological behavior and DSC.

Rheological measurements. - Because the type and shape of aggregates, concentration, and interactions between dispersed droplets in microemulsion systems strongly affect their rheological properties, viscosity measurements were used to confirm conductivity results and to obtain important information concerning structural transformations in the investigated systems (28). The dependence of $\eta$ on $\Phi_{\mathrm{w}}$ along the dilution line $S_{\text {mix }}: \mathrm{O}=8: 2$ of the investigated systems is shown in Figs. $3 \mathrm{c}$ and d. It should be mentioned that in the case of APG-based systems, the real water content values (rather than water fraction added) are plotted against $\eta$ on the respective graphs. Obviously, in comparison with C90/ethoxylated surfactant-TransP/water systems, APG-based systems showed a different rheological behavior, but similar to the microemulsion systems based on sucrose esters (18) and other APGs (5). In the case of APG-containing vehicles, the first part of the curves is missing (Fig. 3c) due to the fact that P2000 and P810 are available as water solutions and, hence, microemulsions with $\Phi_{\mathrm{w}}$ below 18.8 and $14.6 \%(\mathrm{~m} / \mathrm{m})$, respectively, cannot be obtained. As presented in Fig. 3c, the $\eta$ of the microemulsions decreased as $\Phi_{\mathrm{w}}$ increased along the dilution line $S_{\text {mix }}:$ Oil $=8: 2$. A corresponding reduction in the concentration of PG and C90 may explain this effect, since both components offer higher values of $\eta$ than that of water $(1 \mathrm{mPas})(5$, 29). However, in the P810-based system, a sharp change of $\eta$ can be detected at $\Phi_{\mathrm{w}}$ below $23.15 \%(\mathrm{~m} / \mathrm{m})$, whereas for water contents between 23.15 and $48.75 \%(\mathrm{~m} / \mathrm{m}), \eta$ changed slowly, probably due to the gradual move of surfactant molecules from the bulk to the interface to cover water and oil in bicontinuous microemulsions (29). The sudden change of the slope can be referred to the occurrence of microemulsion phase inversion $(26,29,30)$. 
N. Z. Bubić Pajić et al.: Alkyl polyglucoside vs. ethoxylated surfactant-based microemulsions as vehicles for two poorly water-soluble drugs: physicochemical characterization and in vivo skin performance, Acta Pharm. 67 (2017) 415-439.

Thus, this change of $\eta$ at $\Phi_{\mathrm{w}} 23.15 \%(\mathrm{~m} / \mathrm{m})$ indicated the transition from w/o to bicontinuous structure, which correlates well with conductivity results. On the other hand, the respective graph for the system based on P2000 could be divided into three different regions. The first part of the diagram (where $\Phi_{\mathrm{w}}$ is between 18.80 and $26.92 \%, \mathrm{~m} / \mathrm{m}$ ) may be related to the end of the system transition from oil-continuous to bicontinuous, which is in agreement with conductivity measurements. In the second region, when $\Phi_{\mathrm{w}}$ is below $39.10 \%$ $(\mathrm{m} / \mathrm{m})$, a bicontinuous nanostructure was completely developed. As $\Phi_{\mathrm{w}}$ further increased, the bicontinuous structure transformed to the inverted bicontinuous structure, without a fully developed o/w inner structure. Similar rheological behavior of other sugar surfactants was reported earlier (31).

Interestingly, in comparison with APGs, rheological behavior of the vehicles produced using ethoxylated surfactants was found to be very different (Fig. 3d). While $\eta$ of APG-based systems decreased upon dilution with water, in the case of polyoxyethylenes, a rise of $\eta$ was detected at lower water contents. Further addition of water caused a gradual reduction of $\eta$. Further, differences in the rheological behavior between EV and P80based systems can be detected as well. Viscosity in both ethoxylated surfactant-containing systems increased as $\Phi_{\mathrm{w}}$ reached $10 \%(\mathrm{~m} / \mathrm{m})$. This initial increase of $\eta$ could be explained by the attractive interactions and aggregation of water droplets, which include molecular reorganization at the interface (28). Actually, as reported earlier $(28,30)$, transition of spherical droplets to bicontinuous structures is accompanied with a maximum of $\eta$ along with the rise of $\kappa$. Hence, the obtained results suggest that the system structure transformed from oil-continuous (when $\Phi_{\mathrm{w}}$ was less than $10 \%, \mathrm{~m} / \mathrm{m}$ ) to bicontinuous, which is quite consistent with the conductivity results. As $\Phi_{\mathrm{w}}$ became higher than $10 \%(\mathrm{~m} / \mathrm{m})$, the measured values of $\eta$ of the EV-based samples decreased slowly from 22.63 to $16.48 \mathrm{mPas}$, since water and oil in bicontinuous microemulsions became covered with surfactant molecules, which shifted from the bulk to the interface (29). Contrary to the EV-based system, in the P80-containing system, addition of water above $10 \%(\mathrm{~m} / \mathrm{m})$ caused a rise of $\eta$, but the change in this part of the curve was slow. When $\Phi_{\mathrm{w}}$ was between 20 and $50 \%(\mathrm{~m} / \mathrm{m}), \eta$ values were more constant and independent of $\Phi_{\mathrm{w}}$ and then the values slightly decreased until the water content was $60 \%(\mathrm{~m} / \mathrm{m})$. Such a trend was reported earlier $(27,29)$, and this tendency, when $\eta$ of a system becomes higher with water addition, may be ascribed to the increase of water filled channels in a bicontinuous structure (29). The rise of $\eta$ of w/o samples occurs as a consequence of the enhanced attractive interactions between larger dispersed droplet sizes. As $\Phi_{\mathrm{w}}$ is higher, due to the progressive hydration of P80 hydroxyl groups as well as interactions between the interconnected water and oil channels in the bicontinuous region, a maximum of $\eta$ is reached at $50 \%(\mathrm{~m} / \mathrm{m})$ of water. According to this and taking into account the conductivity results obtained for P80 samples, the beginning of phase transition from bicontinuous to o/w microemulsion might be suggested when $\Phi_{\mathrm{w}}$ exceeds $50 \%(\mathrm{~m} / \mathrm{m})$, whereas the w/o nanostructure existed when $\Phi_{\mathrm{w}}$ was below $10 \%(\mathrm{~m} / \mathrm{m})$.

Differential scanning calorimetry (DSC). - DSC cooling curves of APG-based microemulsions (Figs. 4b and c) strongly supported the results of conductivity and rheology measurements. Physical state of water within the system can be investigated depending on the size and position of its freezing peak in the respective DSC cooling curves $(1,2,5)$. Indeed, when water molecules interact strongly, their freezing occurs at lower temperatures (8).

The water phase used in this study showed a sharp, exothermic peak at $-6.92{ }^{\circ} \mathrm{C}$ (Fig. 4 a). DSC measurements of samples produced with P810 are in good agreement with the 
a)

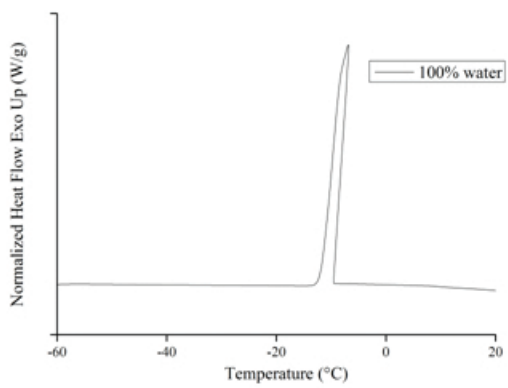

b)

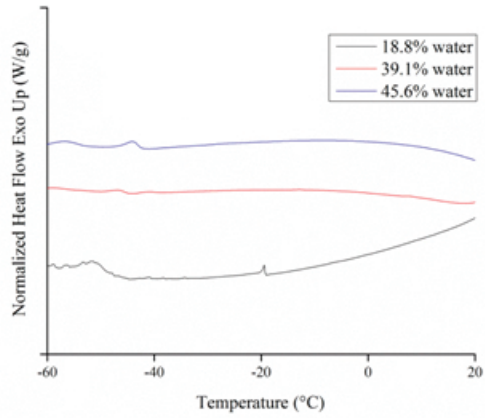

c)

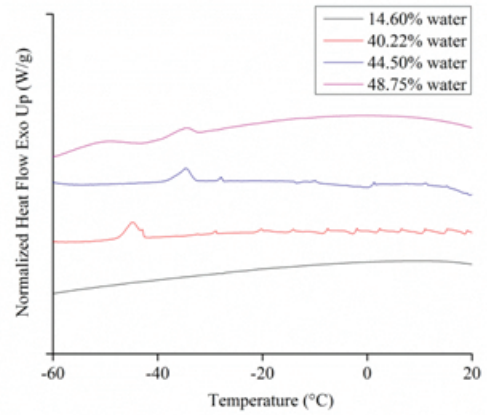

d)

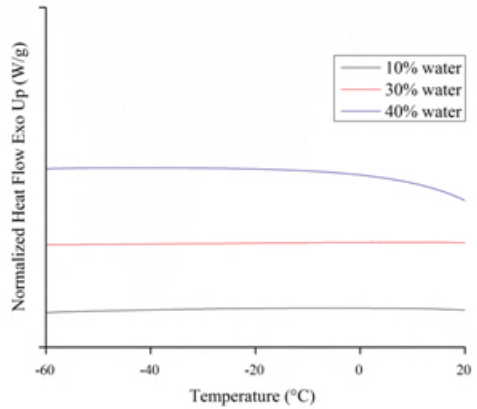

e)

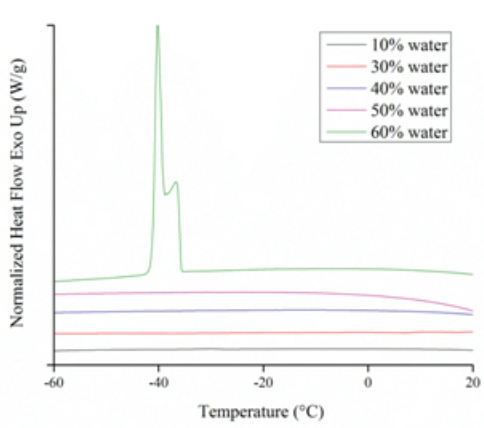

Fig. 4. DSC cooling curves of: a) „bulk” water, b) P2000-, c) P810-, d) EV-, and e) P80-based microemulsion samples along the dilution line $S_{\text {mix }}: \mathrm{O}=8: 2$.

results obtained by conductivity as well as rheological measurements. The cooling curve of sample P810-14.60 (with $\Phi_{\mathrm{w}}$ of $14.60 \%, \mathrm{~m} / \mathrm{m}$ ) did not reveal any freezing peak of water molecules (Fig. 4c), indicating the w/o structure of the microemulsion. One broad exothermic peak at temperatures below $-34{ }^{\circ} \mathrm{C}$ can be observed in the cooling curves of the P810based samples with higher water content, as well as in the cooling curves of the P2000containing pseudo-ternary mixtures (Fig. 4b), indicating bound or interfacial water in these formulations. Due to the polar nature and the presence of hydroxyl groups in the surfactant and cosurfactant structure, water interacted strongly with them, causing a shift 
N. Z. Bubić Pajić et al.: Alkyl polyglucoside vs. ethoxylated surfactant-based microemulsions as vehicles for two poorly water-soluble drugs: physicochemical characterization and in vivo skin performance, Acta Pharm. 67 (2017) 415-439.

of the water freezing point towards lower temperatures and a reduction in freezing enthalpy $(2,18,29)$. In addition, freezing could also be influenced by cosurfactant PG itself, since it has been shown that water-soluble polyols act as cryoprotectants $(2,5)$. In both APGs-based systems, as the water content gets higher, the peak shifts towards higher temperatures. Hence, the water in these microemulsions seems to be involved in hydrating surfactant molecules and, accordingly, the bicontinuous type of structure is presumable along the selected dilution line.

Figs. $4 \mathrm{~d}$ and e represent cooling curves of the EV and P80 containing systems, respectively. Surprisingly, in the curves obtained for the systems based on ethoxylated surfactants, the absence of freezing events was observable for the EV containing samples (even with $\Phi_{\mathrm{w}}$ of $40 \%, \mathrm{~m} / \mathrm{m}$ ) (Fig. $4 \mathrm{~d}$ ) and for the P80-based microemulsions with $\Phi_{\mathrm{w}} 10-50 \%$ $(\mathrm{m} / \mathrm{m})$ as well (Fig. 4e). Thus, it would be reasonable to assume that these samples contain a non-freezable aqueous phase in w/o microemulsions $(19,30)$. However, the presence of free water, without interactions present in the systems, is not expected, since the investigated microemulsions contain a large amount of $S_{\text {mix }}$ and interactions cannot be neglected $(8,30)$. Due to hydroxyl groups in the structure of EV and P80, hydrogen bonds with water molecules might be suggested and, as a consequence, the water freezing point moved towards low temperatures, probably out of the detected range $(2,8,30)$. The only sample that manifested an exothermic peak at $-40.4{ }^{\circ} \mathrm{C}$ was the P80-based vehicle, which has high water content $(60 \%, \mathrm{~m} / \mathrm{m})$ (Fig. $4 \mathrm{e})$. Since concentration of $S_{\text {mix }}$ decreased, the interactions became progressively weaker and water molecules could migrate to the outer phase, which was manifested in a sharp peak that deviated in temperature from that of water, implying loosely interacting water (30). Hence, the appearance of such a peak in the curve representing the P80-60 microemulsion sample may indicate the phase transition from bicontinuous to o/w structure, which is in good agreement with the conductivity and viscosity results.

\section{Solubilization capacity along the selected dilution line}

Solubilization capacity study was performed along the selected water dilution line $S_{\text {mix }}: \mathrm{O}=8: 2$. It was shown in the APG-based systems that complete solubilization of ADA therapeutic concentration was achieved in mixtures of both surfactants, P2000 and P810, with PG as a cosurfactant and C90 as the oil phase. As dilution progressed, loading capacity of the system was not changed: ADA was completely dissolved in all formulations along the selected water dilution line. It is reasonable to suppose that these results could be a consequence of the inherent solubilization potential of the system in parallel with high $\mathrm{pH}$ values caused by the presence of APGs, since the solubility of ADA, as a weak acidic compound, is $\mathrm{pH}$ dependent. In addition, as ADA contains a large apolar domain as well as a carboxyl group as a small polar part of the molecule (Table I), its amphiphilic nature could be presumed and, thus, the solubilized drug could act as a cosurfactant. However, no behavior typical for mesogenic molecules was confirmed for ADA, at least by the results obtained by interfacial tension measurements. Calculated values of equilibrium interfacial tension between water and C90, with and without ADA, were $5.49 \pm 0.06$ and $4.63 \pm 0.33 \mathrm{mN} \mathrm{m}^{-1}$, respectively, indicating that ADA had no amphiphilic properties, although the influence of ADA on interfacial film properties was slight. However, it should be noticed that the surface activity of drugs can be highly affected by changes of system microenvironment (e.g., $\mathrm{pH}$ changes, addition of surface active compounds or lipids). Hence, more detailed investigations concerning surface activity of the drug are required, as well as assessment of the exact 
N. Z. Bubić Pajić et al.: Alkyl polyglucoside vs. ethoxylated surfactant-based microemulsions as vehicles for two poorly water-soluble drugs: physicochemical characterization and in vivo skin performance, Acta Pharm. 67 (2017) 415-439.

locus of the solubilized drug within the formulations (e.g., by using electron paramagnetic resonance spectroscopy), which will be the topic of our further study. However, considering ADA solubility in the microemulsion constituents (Table I), the probability of ADA incorporation into the interfacial film rather than into the oil or water phase (as parent or ionized molecule) becomes higher $(3,15,18)$. Since a total amount of water that could be added in the mixture of P2000, PG and C90 along the dilution line $S_{\text {mix }}: \mathrm{O}=8: 2$ was around $33 \%(\mathrm{~m} / \mathrm{m})$, microemulsion with $25 \%(\mathrm{~m} / \mathrm{m})$ of water added (making $39.1 \%(\mathrm{~m} / \mathrm{m})$ of water in total) was selected for further investigations (the formulation was named APG-1). In order to estimate the influence of APG type on vehicle properties, the P810-based formulation (named APG2) was chosen in such a way that it had almost the same total amount of all ingredients (Table II). However, it was not possible to dissolve SN in APG-based formulations along the selected water dilution line although the solubility of SN in APG-based microemulsion constituents was higher than the solubility of ADA (Table I). These results were expected taking into account the high therapeutic concentration of the drug $(2 \%, \mathrm{~m} / \mathrm{m})$.

Further, the solubilization capacity of both drugs in the systems based on ethoxylated surfactants was investigated. Interestingly, in the C90/EV-TransP/water system, an increase in water content above $30 \%(\mathrm{~m} / \mathrm{m})$ was accompanied by the formation of drug precipitate in the case of both drugs when used in their therapeutic concentrations. ADA $(0.1 \%, \mathrm{~m} / \mathrm{m})$ was solubilized in the formulations with water content up to $30 \%(\mathrm{~m} / \mathrm{m})$, despite its low solubility in the microemulsion constituents, possibly because of its low concentration in parallel with the $\mathrm{pH}$ value of the system. As the measured $\mathrm{pH}$ values (Table II) of sample C90/EV-TransP/water (named ME-ETP) were higher than the $\mathrm{pK}_{\mathrm{a}}$ value of ADA (4.23) (14), it was expected that ADA solubility would be facilitated with increasing the sample $\mathrm{pH}$. On the other hand, in spite of the 20 times higher therapeutic concentration, the solubility of SN in the oil, surfactant and cosurfactant was much higher, leading to its complete dissolution in the microemulsions. By measuring saturation solubility along the selected dilution line $S_{\text {mix }}: \mathrm{O}=8: 2$ (Fig. 5a), we tried to better understand this phenomenon of low ability of this system to solubilize both drugs. The same phenomenon of low solubilization capacity for both ADA and SN and the same trend of dependence of saturated solubility on the water content in the system were interesting due to differences in the structures of model drugs as well as solubility potential in microemulsion components (see Table I). Indeed, at lower contents of water, there was enough oil and amphiphiles to dissolve SN and ADA. As dilution progressed, the content of oil together with $S_{\text {mix }}$ decreased, resulting in reduced saturated solubility values for the drugs. A pronounced decrease in saturation solubility of both drugs was detected at $\Phi_{w^{\prime}}$ up to $10 \%(\mathrm{~m} / \mathrm{m})$, whereas it was gradual in the second region $(10-40 \%, \mathrm{~m} / \mathrm{m})$. Taking into account that the reduction in the saturation solubility of drugs may indicate structural changes caused by dilution $(28,29)$, it could be assumed that the phase transition from w/o into bicontinuous microemulsions occurred when the concentration of the aqueous phase was around $5 \%(\mathrm{~m} / \mathrm{m})$. The transition point in the C90/EV-TransP/water system, defined by the study of saturation capacity for both model drugs, is quite consistent with the conductivity and rheology measurements, indicating that a structural transformation of the microemulsion system occurred. In the mixtures free of water, the interface of the reverse micelles solubilizes higher amounts of the drugs than in the presence of water, which may be attributable to the interfacial locus of the drugs $(15,30)$. Addition of water to the mixture of $S_{\text {mix }}$ and oil induced the formation of w/o microemulsions. In fact, incorporation of water into the systems leads to interactions between the hydrophilic groups of surfactant and water, thus hydrating the surfac- 
a)

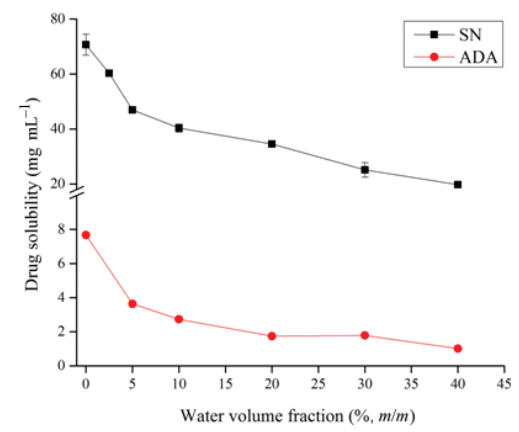

b)

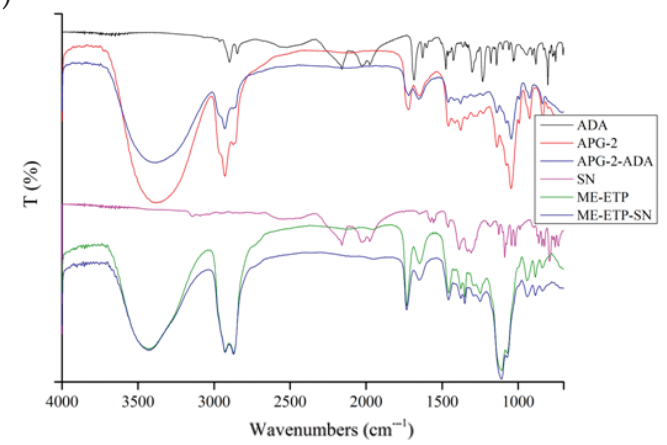

Fig. 5. a) Adapalene and sertaconazole nitrate saturation solubility in the C90/EV-Trans $\mathrm{P} /$ water system along the dilution line $S_{\text {mix }}: \mathrm{O}=8: 2$ as a function of the water volume fraction, b) FTIR spectra of pure ADA, APG-2, APG-2-ADA, pure SN, ME-ETP and ME-ETP-SN.

tant molecules and decreasing the interfacial area. As a consequence, there were fewer surfactant molecules available at the interface for interactions with both drugs, leading to their lower solubility in the formulations (29) (the first region, Fig. 5a). Upon further dilution (the second region, Fig. 5a), the solubilization capacity for both drugs slightly decreased due to the formation of bicontinuous domains $(28,30)$.

Surprisingly, despite higher solubility in EV than in P80, a similar loading capacity for $\mathrm{SN}$ was observed in the presence of the other investigated ethoxylated surfactant (P80), whereas solubilization capacity for ADA in this system was rather low. To be more precise, the obtained solubility values in system components (Table I) considered together with the structure of drugs may suggest that the largest fraction of SN in the P80-based system at a low water content could be partitioned between the interface and the oil phase. In fact, the $\mathrm{SN}$ solubilization capacity along the investigated water dilution line $S_{\text {mix }}: \mathrm{O}=8: 2$ in the C90/P80-TransP/water system reduced at water fractions higher than $30 \%(\mathrm{~m} / \mathrm{m})$, and this was accompanied by the formation of drug precipitate (Fig. 2d, inset). Lower solubility of $\mathrm{SN}$ was somehow expected as the progressive dilution of the investigated system was followed by a decrease of the content of oil along with $S_{\text {mix }}$.

Finally, taking all together, beside formulations APG-1 and APG-2, we have also selected ME-ETP as a vehicle for topical application of ADA, three of them in total (Table II). As the solubility of ADA in P80 was found to be the lowest among the surfactants investigated, lower loading capacity of P80 microemulsions than that of EV was an expected consequence. Moreover, since complete solubilization of ADA was achieved in the P80based system containing maximum $15 \%(\mathrm{~m} / \mathrm{m})$ of water, this formulation was excluded from further characterization due to the higher amount of surfactant and cosurfactant compared to the previously selected APG- and EV-based vehicles. For SN, only two microemulsion formulations containing the same concentration of components were selected for topical application (ME-ETP and ME-P). Overall, such a selection of investigated vehicles offered the possibility to investigate the effects of different surfactants on the microemulsion characteristics, which at the same time may have an influence on ADA and SN skin delivery (Table II). 
N. Z. Bubić Pajić et al.: Alkyl polyglucoside vs. ethoxylated surfactant-based microemulsions as vehicles for two poorly water-soluble drugs: physicochemical characterization and in vivo skin performance, Acta Pharm. 67 (2017) 415-439.

Table II. Composition and characteristics of selected microemulsion formulations ${ }^{\mathrm{a}}$

\begin{tabular}{|c|c|c|c|c|c|c|c|}
\hline Formulation & $\begin{array}{l}\text { Compos } \\
\text { of unloa } \\
\text { formula } \\
\%(\mathrm{~m} / \mathrm{m})\end{array}$ & $\begin{array}{l}\text { tion } \\
\text { led } \\
\text { lons }\end{array}$ & $\mathrm{pH}$ & $\begin{array}{c}\text { Corrected } \\
\mathrm{pH}\end{array}$ & $\begin{array}{l}\text { Original } \kappa \\
\left(\mu S \mathrm{~cm}^{-1}\right)\end{array}$ & $\begin{array}{c}\kappa \text { after } \mathrm{pH} \\
\text { adjustment } \\
\left(\mu S \mathrm{~cm}^{-1}\right)\end{array}$ & $\begin{array}{c}\eta \\
(\mathrm{mPa} s)\end{array}$ \\
\hline APG-1 & $\mathrm{C} 90$ & 15.0 & & & & & \\
\hline unloaded & $\begin{array}{l}\text { P2000 } \\
\text { PG }\end{array}$ & $\begin{array}{l}15.9 \\
30.0\end{array}$ & $8.50 \pm 0.06$ & $7.88 \pm 0.04$ & $729.0 \pm 5.0$ & $902.0 \pm 12.0$ & $27.86 \pm 0.30$ \\
\hline ADA-loaded & Water & 39.1 & $8.49 \pm 0.01$ & $7.67 \pm 0.03$ & $711.3 \pm 0.7$ & $836.0 \pm 7.0$ & $27.90 \pm 0.32$ \\
\hline APG-2 & C90 & 14.0 & & & & & \\
\hline unloaded & $\begin{array}{l}\text { P810 } \\
\text { PG }\end{array}$ & $\begin{array}{l}17.8 \\
28.0\end{array}$ & $8.77 \pm 0.07$ & $6.85 \pm 0.03$ & $834.7 \pm 1.3$ & $1056.3 \pm 7.0$ & $25.31 \pm 1.10$ \\
\hline ADA-loaded & Water & 40.2 & $8.78 \pm 0.02$ & $6.72 \pm 0.02$ & $763.7 \pm 0.7$ & $1022.0 \pm 14.0$ & $26.81 \pm 0.19$ \\
\hline ME-ETP & & & & & & & \\
\hline unloaded & C90 & 14.0 & $7.47 \pm 0.06$ & - & $62.5 \pm 1.0$ & - & $18.84 \pm 1.43$ \\
\hline ADA-loaded & $\begin{array}{l}\text { EV } \\
\text { TransP }\end{array}$ & $\begin{array}{l}28.0 \\
28.0\end{array}$ & $7.38 \pm 0.01$ & - & $61.6 \pm 0.6$ & - & $19.16 \pm 0.27$ \\
\hline SN-loaded & Water & 30.0 & $4.39 \pm 0.04$ & - & $268.0 \pm 2.0$ & - & $21.91 \pm 1.71$ \\
\hline ME-P & $\mathrm{C} 90$ & 14.0 & & & & & \\
\hline unloaded & $\begin{array}{l}\text { P80 } \\
\text { TransP }\end{array}$ & $\begin{array}{l}28.0 \\
28.0\end{array}$ & $4.42 \pm 0.03$ & - & $48.5 \pm 1.1$ & - & $48.79 \pm 1.17$ \\
\hline SN-loaded & Water & 30.0 & $3.67 \pm 0.11$ & - & $242.3 \pm 1.2$ & - & $51.01 \pm 0.97$ \\
\hline
\end{tabular}

${ }^{a}$ Results are expressed as mean \pm SD of triplicate experiments.

\section{Physicochemical characterization of the selected microemulsion samples}

The investigated microemulsion vehicles and the corresponding drug-loaded samples were homogeneous and transparent liquids at room temperature. When observed under a cross polarizer, all microemulsions appeared completely dark, which indicated their homogeneity and optical isotropy (Fig. 2b inset). No traces of undissolved drugs were seen in the drug-loaded formulations. The appearance of selected microemulsions did not change during one year-storage at room temperature. In order to anticipate the possible nanostructure of the selected samples from Table II and to further characterize selected formulations, it is necessary to perform comprehensive physicochemical characterization of the selected unloaded and drug-loaded microemulsions. The $\mathrm{pH}$ values of APG-based microemulsions were found to be above 8 (Table II). In comparison with the other polyglucoside-based formulations $(7,9)$, these values fall within a similar range. Incorporation of ADA did not significantly affect the observed $\mathrm{pH}$ values of the formulations. Since healthy skin has $\mathrm{pH}$ in the range 4-6 (9), and formulations displaying $\mathrm{pH}$ closer to these values are generally considered well-tolerated, the $\mathrm{pH}$ was adjusted as much as possible below 8 for further evaluation, using $0.35 \%(\mathrm{~m} / \mathrm{m})$ phosphoric acid (Table II). Further, by reducing the $\mathrm{pH}$ value, the unionized/ionized ADA ratio should increase, which can be beneficial for drug delivery, as penetration of unionized species into skin is often favored. Further addi- 
N. Z. Bubić Pajić et al.: Alkyl polyglucoside vs. ethoxylated surfactant-based microemulsions as vehicles for two poorly water-soluble drugs: physicochemical characterization and in vivo skin performance, Acta Pharm. 67 (2017) 415-439.

tion of the acid resulted in occurrence of turbidity, probably due to the precipitation of ADA (confirmed using polarization microscopy). Although the obtained solubility results favored the interfacial film as the main locus for solubilization of the majority of drug molecules, it is probable that some ADA molecules are present in the water phase. The latter assumption is supported by the fact that $\mathrm{pH}$ values of drug-loaded formulations are lower than the values of placebo samples. However, as $\mathrm{pH}$ values of formulations became lower, the number of unionized ADA molecules was higher, saturating the interfacial area, which led to drug precipitation. Also, addition of an electrolyte could alter the interfacial film properties $(15,24,29)$ in such a way as to enhance penetration of oil at the interface instead of drug molecules, which could also contribute to drug precipitation. Contrary to APG-based microemulsions, the formulations based on EV and P80 had pH values appropriate for topical application. However, contrary to ADA, incorporation of SN strongly affected the observed $\mathrm{pH}$ values of the microemulsions (Table II). Decreased $\mathrm{pH}$ values, after loading SN, can be ascribed to the strong acidic character of the nitrate anions predominantly partitioned into the water phase of the system. Nevertheless, the $\mathrm{pH}$ of SNloaded formulations was acceptable for dermal application.

According to the results of electrical conductivity, rheology and DSC measurements, for all the selected microemulsion formulations, at ascending parts of the conductivity plots (Figs. 3a and b), bicontinuous structure is suggested. SN incorporation strongly affected the $\kappa$ values of microemulsion vehicles (Table II), increasing the measured $\kappa$ values of drug loaded formulations by about 4 and 11 times (for EV- and P80-based formulations, respectively). This can be explained by the fact that conductivity increased because nitrate anions passed through water channels, formed by merging of water droplets. In contrast, the incorporation of ADA into microemulsion vehicles led to a small decrease in $\kappa$ (Table II), indicating that incorporation of the drug did not affect the nanostructure of the system (28).

Shear stress $(\tau)$ was found to be linearly proportional to the shear rate $(\gamma)$, while $\eta$ was independent of $\gamma$, indicating that studied microemulsions had Newtonian flow behavior without thixotropy (data not shown). These results are in accord with the earlier observation that microemulsions generally exhibit this flow type $(2,7,18)$. Incorporation of the drugs in microemulsion vehicles affected their $\eta$ (Table II), while the flow behavior remained unchanged. The $\eta$ values of the drug-loaded microemulsions were higher than those obtained for blank vehicles. This effect was slightly more pronounced after SN addition, which may be attributed to its higher concentration in the formulations. Due to their structures and solubility in microemulsion constituents, the most significant locus for solubilization of both ADA and SN is the interfacial film, which contributes to higher $\eta$ of bicontinuous microemulsion systems. However, no statistically significant difference of $\eta$ values was observed between drug-loaded and blank vehicles.

Fig. $5 \mathrm{~b}$ represents the FTIR spectra of the selected unloaded and drug-loaded microemulsions, as well as of pure ADA and SN. The FTIR spectra of ADA (Fig. 5b) show a characteristic stretch between 3000 and $2800 \mathrm{~cm}^{-1}\left(\mathrm{O}-\mathrm{H}\right.$ stretch in acid), a stretch at $1686 \mathrm{~cm}^{-1}$ ( $C=\mathrm{O}$ stretch in acid) while aromatic groups were found at $900-650 \mathrm{~cm}^{-1}$. In the case of SN (Fig. 5b), the S-H and C-N bonds were found at 2543 and $1380 \mathrm{~cm}^{-1}$, respectively. The benzene ring was detected at 1530-1660, the $C=C$ stretch at 1459 , the $C-H$ stretch at 3144 and the C-O group at $1300-1000 \mathrm{~cm}^{-1}$. After loading microemulsions with ADA or SN, no specific peaks corresponding to the drugs could be observed in the FTIR spectra, as they were buried in the peaks of other microemulsion constituents. In addition, there were no new peaks, indicating the absence of chemical interactions after incorporation of either model drug (32). 
In vivo skin performance of selected microemulsions

Dermal application of pharmaceutics is usually associated with an already impaired skin barrier function and, therefore, it is of the paramount importance to have a vehicle with low or without any potential for skin irritation. Based on the microemulsion composition, with a predominantly high surfactant concentration, they are obviously considered as vehicles with a certain skin irritation potential and in order to investigate it, in vivo assessment was performed.

Results obtained in our 24-hour occlusion study are presented in Fig. 6 for all the measured parameters (EI, TEWL and SCH) for selected samples, APG-1, APG-2, ME-ETP and ME-P, as well as untreated controls with (NCO) and without occlusion (NC). Parameters are expressed as the absolute change of values $3 \mathrm{~h}$ after occlusion and initial (baseline) value, for each volunteer with the mean value for each test site (Fig. 6).

a)

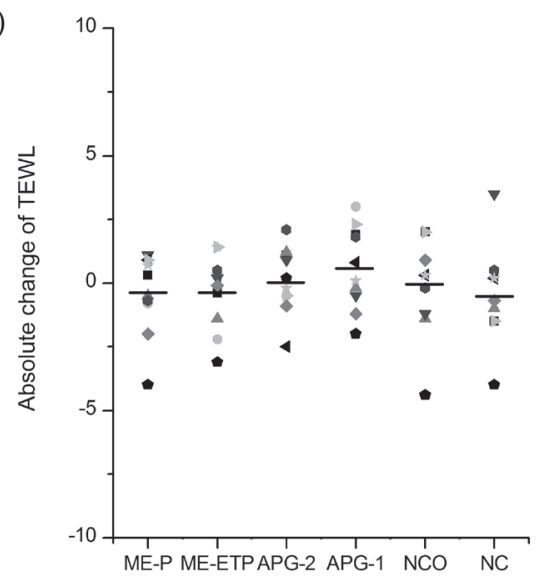

c)

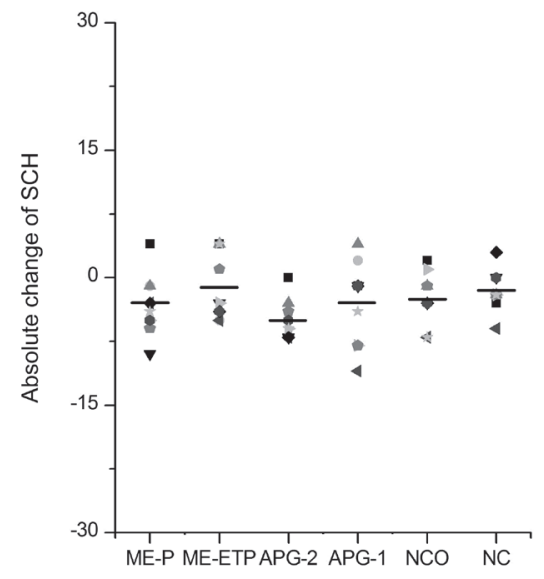

b)

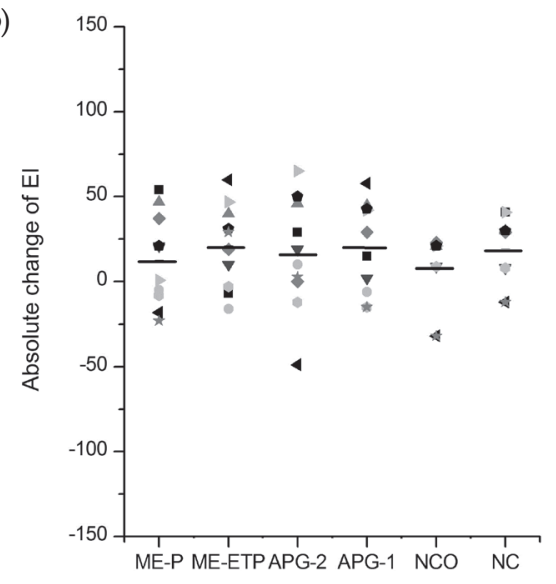

Fig. 6. Absolute changes of: a) transepidermal water loss - TEWL, b) erythema index - EI, and c) stratum corneum hydration $\mathrm{SCH}$; Absolute changes for each volunteer (obtained as differences between the values gained $3 \mathrm{~h}$ after occlusion removal and initial values) with mean values (long dash). 
N. Z. Bubić Pajić et al.: Alkyl polyglucoside vs. ethoxylated surfactant-based microemulsions as vehicles for two poorly water-soluble drugs: physicochemical characterization and in vivo skin performance, Acta Pharm. 67 (2017) 415-439.

For selected samples, no statistically significant changes were obtained for any investigated parameter related to their baselines and for both non-treated controls; only a slight trend of increase could be observed for all EI values except untreated controls, but due to the large interindividual variations between results, along with the intact barrier function (TEWL values remained unchanged) after the occlusion test, these trends are not considered important.

In other words, the investigated microemulsions did not induce redness or skin barrier impairment, nor had any effect on skin hydration levels. Obtained results indicate satisfying preliminary safety profiles of formulations based on APGs (P2000 and P810) as well as on ethoxylated surfactants (EV and P80). In this regard, it is important to emphasize that there was no statistically significant difference between all the tested formulations regarding the changes in monitored skin parameters in the current limited number of human volunteers. However, it should be noted that the aim of this study, which included 10 healthy participants, was a preliminary investigation of the skin irritation potential of the developed microemulsion formulations. Thus, a more comprehensive chronic study needs to be performed in order to assess their influence on the skin regarding irritation as well as histopathological changes.

Finally, it should be pointed out that although in vivo screening of the microemulsion irritation profile revealed good safety profiles of all investigated systems, sugar based surfactants, such as APGs, seem to be superior over conventionally used tensides regarding topical drug delivery (2), which may be considered as an advantage of sugar-based surfactants over the conventional emulsifier. Also, taking into account the specific requirements of modern patients for "green" products, the use of surfactants of natural origin (such as APGs and, according to the manufacturer, EV as well), which are skin- and environmental-tolerable, better patient compliance could be achieved. Hence, further investigations should be performed in order to examine their potential to deliver both selected drugs (ADA and SN) at the target site of action in the skin.

\section{CONCLUSIONS}

The main findings of our research, intended to develop biocompatible tailor-made microemulsion vehicles for two model drugs (ADA and SN) strongly differing in their therapeutic concentrations and taking into account the nature of employed surfactants, can be summarized as follows:

The tail length and HLB value of used APGs significantly affected the formulated microemulsion area boundaries; namely, extension of the single-phase microemulsion area was observed when APG with a shorter tail length and higher HLB value was used.

In both ethoxylated surfactants, wider microemulsion areas were detected, but in the case of P80, the surfactant with higher HLB and longer chain length, this phenomenon was more pronounced; nonetheless, all systems changed in their nanostructures upon dilution with water, showing the above elaborated surfactant-dependent specific phase transitions.

$\kappa, \eta$ and $\mathrm{pH}$ of the vehicles were strongly affected by SN incorporation, while these properties remained almost unchanged after ADA incorporation, irrespective of the surfactant used. 
N. Z. Bubić Pajić et al.: Alkyl polyglucoside vs. ethoxylated surfactant-based microemulsions as vehicles for two poorly water-soluble drugs: physicochemical characterization and in vivo skin performance, Acta Pharm. 67 (2017) 415-439.

Regarding the screening of their in vivo skin irritation profiles, all selected microemulsions proved to be mild to the skin, irrespective of the type of the surfactant used, since no cutaneous adverse effects were observed throughout the study.

Somehow expected, each of the investigated drugs required specific development of a tailor-made microemulsion vehicle; for example, APG-based formulations, containing less than $20 \%(\mathrm{~m} / \mathrm{m})$ of the surfactant, were appropriate carriers for ADA.

Even so, it appeared that the microemulsion system based on EV (glycereth-7-caprylate/caprate), containing also C90 (propylene glycol monocaprylate) and TransP (highly purified diethylene glycol monoethyl ether) with $30 \%$ of water was suitable for both model drugs, irrespective of their specificities.

Nevertheless, it should be emphasized that to finally prove the functionality of each of the surfactants for formulation of a microemulsion as the vehicle for a given drug, further exploration of the system capability to deliver it effectively should be performed.

Acknowledgment. - The authors acknowledge the financial support from the Ministry of Education, Science and Technological Development, Republic of Serbia, through Project TR34031. The authors are grateful to BASF for supplying alkyl polyglucosides, Kao Chemicals Europe for their kind donation of Emanon EV-E, Gattefosse SAS for free samples of Capryol 90 and Transcutol P and the Agency for Medicinal Products and Medical Devices, Bosnia and Herzegovina, for their kind donation of adapalene reference standard.

\section{REFERENCES}

1. S. Heuschkel, A. Goebel and R. H. H. Neubert, Microemulsions - Modern colloidal carrier for dermal and transdermal drug delivery, J. Pharm. Sci. 97 (2008) 603-631; http://dx.doi.org/10.1002/ jps.20995

2. A. Goebel, U. Knie, C. Abels, J. Wohlrab and R. Neubert, Dermal targeting using colloidal carrier systems with linoleic acid, Eur. J. Pharm. Biopharm. 75 (2010) 162-172; https://doi.org/10.1016/j. ijpharm.2010.11.029

3. M. J. Lawrence and G. D. Rees, Microemulsion-based media as novel drug delivery systems, Adv. Drug Deliv. Rev. 64 (2012) 175-193; https://doi.org/10.1016/j.addr.2012.09.018

4. K. Fukuda, U. Olsson and M. Ueno, Microemulsion formed by alkyl polyglucoside and an alkyl glycerol ether with weakly charged films, Colloids Surf. B. 20 (2001) 129-135; https://doi.org/10.1016/ S0927-7765(00)00183-1

5. A. Goebel, R. Neubert and J. Wohlrab, Dermal targeting of tacrolimus using colloidal carrier systems, Int. J. Pharm. 404 (2011) 159-168; https://doi.org/10.1016/j.ejpb.2010.02.001

6. W. von Rybinski and K. Hill, Alkyl Polyglycosides - properties and applications of a new class of surfactants, Angew. Chem. Int. E. Engl. 37 (1998) 1328-1345; http://dx.doi.org/10.1002/(SICI)15213773(19980605)37:10<1328::AID-ANIE1328

7. A. N. El Meshad and M. I. Tadros, Transdermal delivery of an anti-cancer drug via w/o emulsions based on alkyl polyglucosides and lecithin: design, characterization, and in vivo evaluation of the possible irritation potential in rats, AAPS PharmSciTech. 12 (2011) 1-9; https://doi.org/10.1208/ s12249-010-9557-y

8. A. Graf, E. Ablinger, S. Peters, A. Zimmer, S. Hook and T. Rades, Microemulsions containing lecithin and sugar-based surfactants: Nanoparticle templates for delivery of proteins and peptides, Int. J. Pharm. 350 (2008) 351-360; https://doi.org/10.1016/j.ijpharm.2007.08.053 
N. Z. Bubić Pajić et al.: Alkyl polyglucoside vs. ethoxylated surfactant-based microemulsions as vehicles for two poorly water-soluble drugs: physicochemical characterization and in vivo skin performance, Acta Pharm. 67 (2017) 415-439.

9. A. Cichewicz, C. Pacleb, A. Connors, M. A. Hass and L. B. Lopes, Cutaneous delivery of $\alpha$-tocopherol and lipoic acid using microemulsions: influence of composition and charge, J. Pharm. Pharmacol. 65 (2013) 817-826; https://doi.org/10.1111/jphp.12045

10. D. Pepe, J. Phelps, K. Lewis, J. DuJack, K. Scarlett, S. Jahan, E. Bonnier, T. Milic-Pasetto, M. A. Hass and L. B. Lopes, Decylglucoside-based microemulsions for cutaneous localization of lycopene and ascorbic acid, Int. J. Pharm. 434 (2012) 420-428; https://doi.org/10.1016/j.ijpharm.2012.06.016

11. A. J. Jain, A. Jain, N. K. Garg, A. Agarwal, A. Jain, S. A. Jain, R. K. Tyagi, R. K. Jain, H. Agrawal and G. P. Agrawal, Adapalene loaded solid lipid nanoparticles gel: an effective approach for acne treatment, Colloids Surf. B. 12 (2014) 222-229; https://doi.org/10.1016/j.colsurfb.2014.05.041

12. M. M. Abdellatif, I. A. Khalil and M. A. F. Khalil, Sertaconazole nitrate loaded nanovesicular systems for targeting skin fungal infection: in-vitro, ex-vivo and in-vivo evaluation, Int. J. Pharm. 527 (2017) 1-11; https://doi.org/10.1016/j.ijpharm.2017.05.029

13. S. Güngör, M. S. Erdal and B. Aksu, New formulation strategies in topical antifungal therapy, JCDSA. 3 (2013) 56-65; http://dx.doi.org/10.4236/jcdsa.2013.31A009

14. G. Bhatia, Y. Zhou and A. K. Banga, Adapalene microemulsion for transfollicular drug delivery, J. Pharm. Sci. 102 (2013) 2622-2631; https://doi.org/10.1002/jps.23627

15. A. S. Narang, D. Delmarre and D. Gao, Stable drug encapsulation in micelles and microemulsions, Int. J. Pharm. 345 (2007) 9-25; https://doi.org/10.1016/j.ijpharm.2007.08.057

16. M. Miastkowska, M. Banach, J. Pulit-Prociak, E. Sikora, A. Glogowska and M. Zielina, Statistical analysis of optimal ultrasound emulsification parameters in thistle-oil nanoemulsions, J. Surf. Deterg. 20 (2017) 233-246; https://doi.org/10.1007/s11743-016-1887-7

17. C. M. Keck, A. Kovacevic, R. H. Müller, S. Savic, G. Vuleta and J. Milic, Formulation of solid lipid nanoparticles (SLN): The value of different alkyl polyglucoside surfactants, Int. J. Pharm. 474 (2014) 3-41; https://doi.org/10.1016/j.ijpharm.2014.08.008

18. M. N. Todosijevic, N. D. Cekic, M. M. Savic, M. Gasperlin, D. V. Randjelovic and S. D. Savic, Sucrose ester-based biocompatible microemulsions as vehicles for aceclofenac as a model drug: formulation approach using D-optimal mixture design, Colloid Polym. Sci. 292 (2014) 3061-307; https://doi.org/10.1007/s00396-014-3351-4

19. J. Zhang and B. Michniak-Kohn, Investigation of microemulsion microstructures and their relationship to transdermal permeation of model drugs: ketoprofen, lidocaine, and caffeine, Int. J. Pharm. 421 (2011) 34-44; https://doi.org/10.1016/j.ijpharm.2011.09.014

20. E. Berardesca, EEMCO guidance for the assessment of stratum corneum hydration: electrical methods, Skin Res. Tech. 3 (1997) 126-132; http://dx.doi.org/10.1111/j.1600-0846.1997.tb00174.x

21. P. Clarys, K. Alewaeters, R. Lambrecht and A.O. Barel, Skin color measurements: comparison between three instruments: the Chromameter ${ }^{\circledR}$, the DermaSpectrometer ${ }^{\circledR}$ and the Mexameter ${ }^{\circledR}$, Skin Res. Technol. 6 (2000) 230-238; https://doi.org/10.1034/j.1600-0846.2000.006004230.x

22. V. Rogiers, EEMCO guidance for the assessment of transepidermal water loss in cosmetic sciences, Skin Pharmacol. Appl. Skin Physiol. 14 (2001) 117-128; http://dx.doi.org/10.1159/000056341

23. L. D. Ryan and E. W. Kaler, Alkyl polyglucoside microemulsion phase behaviour, Colloids Surf. A. 176 (2001) 69-83; https://doi.org/10.1016/S0927-7757(00)00614-2

24. M. Fanun, Phase behavior, transport, diffusion and structural parameters of nonionic surfactants microemulsions, J. Mol. Liq. 139 (2008) 14-22; https://doi.org/10.1016/j.molliq.2007.10.005

25. L. Djekic, M. Primorac and J. Jockovic, Phase behaviour, microstructure and ibuprofen solubilization capacity of pseudo-ternary nonionic microemulsions, J. Mol. Liq. 160 (2011) 81-87; https://doi. org/10.1016/j.molliq.2011.02.010

26. G. Coneac, V. Vlaia, I. Olariu, A. M. Mut, D. F. Anghel, C. Ilie, C. Popoiu, D. Lupuleasa and L. Vlaia, Development and evaluation of new microemulsion-based hydrogel formulations for topical de- 
N. Z. Bubić Pajić et al.: Alkyl polyglucoside vs. ethoxylated surfactant-based microemulsions as vehicles for two poorly water-soluble drugs: physicochemical characterization and in vivo skin performance, Acta Pharm. 67 (2017) 415-439.

livery of fluconazole, AAPS PharmSciTech, 16 (2015) 889-904; https://doi.org/10.1208/s12249-0140275-8

27. S. K. Mehta and K. Bala, Volumetric and transport properties in microemulsions and the point of view of percolation theory, Physical Review E. 51 (1995) 5732-5737.

28. A. Zvonar, B. Rozman, M. Bester Rogac and M. Gasperlin, The influence of microstructure on celecoxib release from a pharmaceutically applicable system: Mygliol $812^{\circledR} /$ Labrasol $^{\circledR} /$ Plurol Oleique $^{\circledR} /$ Water mixtures, Acta Chim. Slov. 56 (2009) 131-138.

29. M. Fanun, Conductivity, viscosity, NMR and diclofenac solubilization capacity studies of mixed nonionic surfactants microemulsions, J. Mol. Liq. 135 (2007) 5-13; https://doi.org/10.1016/j.molliq.2006.09.003

30. F. Podlogar, M. Gasperlin, M. Tomsic, A. Jamnik and M. Bester Rogac, Structural characterisation of water-Tween $40^{\circledR} /$ Imwitor $308^{\circledR}$-isopropyl myristate microemulsions using different experimental methods, Int. J. Pharm. 276 (2004) 115-128; https://doi.org/10.1016/j.ijpharm.2004.02.018

31. M. Fanun, Properties of microemulsions with sugar surfactants and peppermint oil, Colloid Polym. Sci. 287 (2009) 899-910; https://doi.org/10.1007/s00396-009-2043-y

32. R. M. Hathout, T. J. Woodman, S. Mansour, N. D. Mortada, A. S. Geneidi and R. H. Guy, Microemulsion formulations for the transdermal delivery of testosterone, Eur. J. Pharm. Sci. 40 (2010) 188-196; https://doi.org/10.1016/j.ejps.2010.03.008 